\title{
Modelling of Instabilities in Coupled Electrochemical and Biochemical Reaction Systems
}

\author{
Gerold Baier, Sven Sahle, and Peter Urban* \\ Institute for Chemical Plant Physiology, University of Tübingen, Tübingen, FRG
}

Z. Naturforsch. 48 a, 643-645 (1993); received February 16, 1993

A three-cell model of an enzymatic electrocatalytic experiment is introduced. Inhibition of a Michaelis-Menten type of reaction leads to bistability and oscillations under potentiostatic conditions.

\section{Introduction}

An electrochemical reaction followed by a chemical reaction that recovers the electroactive species in its original form is referred to as electrocatalysis. In aqueous solutions at room temperature the recovering can be achieved with the aid of an enzyme as catalysts which converts substrate $\mathrm{S}$ to product $\mathrm{P}$. Figure 1 shows a scheme of such a system for the redox couple OX and RED. This electrochemically mediated synthesis of product $\mathrm{P}$ from substrate $\mathrm{S}$ leads to an increase of the diffusion-limited current under potentiostatic conditions due to reduction of OX. This principle is the basis of amperometric biosensors [1].

The peroxidase catalyzed reduction of hydrogen peroxide (S) with hydroquinone as H-Donor (RED) is an example. This particular system has been shown to oscillate under potentiostatic conditions [2]. Figure 2 shows three responses of this system at three different concentrations of hydrogen peroxide in the initial stock solution. Here we would like to report on attempts to explain the observed dynamic instability of the steady state current in the electrochemical reaction-diffusion system.

\section{An ODE Model}

The dynamics of the electroactive species p-benzoquinone can in a first step be described by the partial

\footnotetext{
* Present address: Daimler Benz AG, Forschungszentrum Ulm, Ulm, FRG.

Reprint requests to $\mathrm{G}$. Baier, Institut für Chemische Pflanzenphysiologie, Universität Tübingen, Corrensstr. 41, W-7400 Tübingen, FRG.
}

differential equation (PDE) with one spatial dimension

$$
\frac{\partial x}{\partial t}=k_{1} \frac{x_{0}-x}{k_{2}+\left(x_{0}-x\right)}+D_{x} \frac{\partial^{2} x}{\partial l^{2}},
$$

where $x$ denotes p-benzoquinone, $x_{0}$ is the initial concentration of benzoquinone, and the peroxidase is assumed to exhibit Michaelis-Menten kinetics. The concentration of hydrogen peroxide is taken to be constant, as in the case of large excess. This latter assumption may correspond to experiments, where single-peak large amplitude oscillations are stable for high concentration of hydrogen peroxide. Simulations of the electrocatalytic experiment in terms of the reaction-diffusion equation with Nernst conditions for reversible electron transfer at the electrode and constant concentration of $x$ inside the solution is then straightforward [4]. However, the single-variable PDE with Michaelis-Menten kinetics is not capable of oscillations under realistic assumptions.

We now take into account that production of each molecule of hydroquinone at the electrode requires

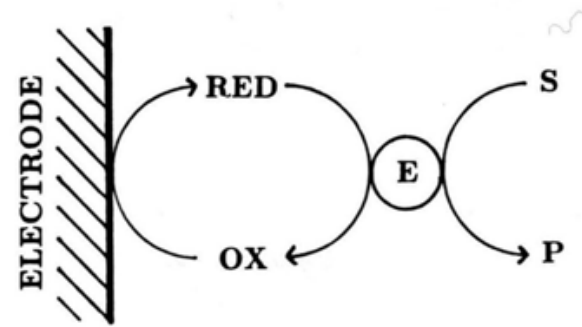

Fig. 1. Principle of the reaction of a substrate $S$ and a second substrate RED catalyzed by an enzyme E (yielding products $\mathrm{P}$ and $\mathrm{OX}$ ) with an electrochemical feedback loop to recover RED from OX at the surface of an electrode. $\mathrm{S}=\mathrm{H}_{2} \mathrm{O}_{2}$, $\mathrm{RED}=$ hydroquinone, $\mathrm{E}=$ horseradish peroxidase, $\mathrm{P}=\mathrm{H}_{2} \mathrm{O}$, $\mathrm{OX}=1,4$-benzoquinone (see [2]).

0932-0784 / $93 / 0500-0643 \$ 01.30 / 0$. - Please order a reprint rather than making your own copy. 


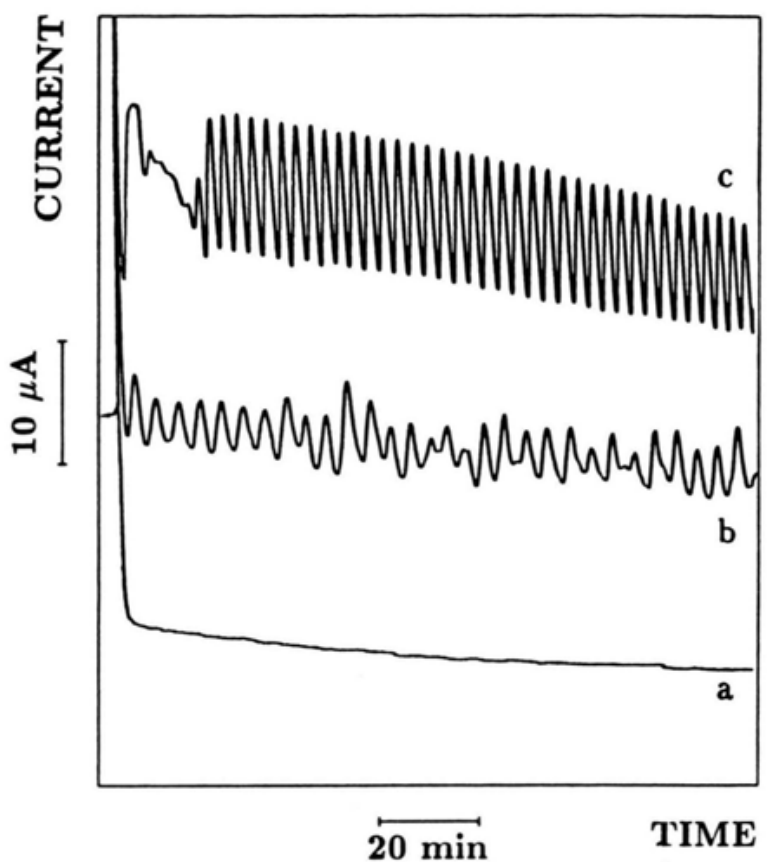

Fig. 2. Time series of current response at a constant potential of $-300 \mathrm{mV}$. Initial concentrations are: [benzoquinone] ${ }_{0}=$ $4 \mathrm{mM}$, [peroxidase] $]_{0}=0.21 \mu \mathrm{M}, 0.1 \mathrm{M}$ acetate buffer, $\mathrm{pH}=$ 5.1. a) $\left[\mathrm{H}_{2} \mathrm{O}\right]_{0}=2 \mathrm{mM}$, b) $\left[\mathrm{H}_{2} \mathrm{O}_{2}\right]_{0}=2.5 \mathrm{mM}$, c) $\left[\mathrm{H}_{2} \mathrm{O}_{2}\right]_{0}$ $=3.0 \mathrm{mM}$. See [3] for details.

two protons. Thus the reduction of benzoquinone causes a decrease of the concentration of protons near the electrode, and $\mathrm{pH}$ increases as the reaction continues. In general, the action of the enzyme, however, is strongly dependent on $\mathrm{pH}$. If the system is started with a solution at $\mathrm{pH}$-optimum of the enzyme, we therefore expect the rate of catalysis to decrease near the electrode as the $\mathrm{pH}$ becomes alkaline. Assuming a decrease of protons, we can model hydroxide ions as inhibitors of the kinetics of benzoquinone:

$$
\frac{\mathrm{d} x}{\mathrm{~d} t}=k_{1} \frac{x_{0}-x}{k_{2}+\left(x_{0}-x\right)+k_{3} y^{a}},
$$

where $y$ denotes the concentration of inhibitor and a simple rational function is introduced for the inhibition.

Inhibitor is produced at the electrode at a rate proportional to the current. It then diffuses into the solution acording to Fick's law and is consumed by protonation from the electrolyte. This yields a second variable of the PDE. Electrochemical diffusion processes can be simulated using the method of finite differences. In that case continuous space and time are approximated by a map lattice with diffusively coupled
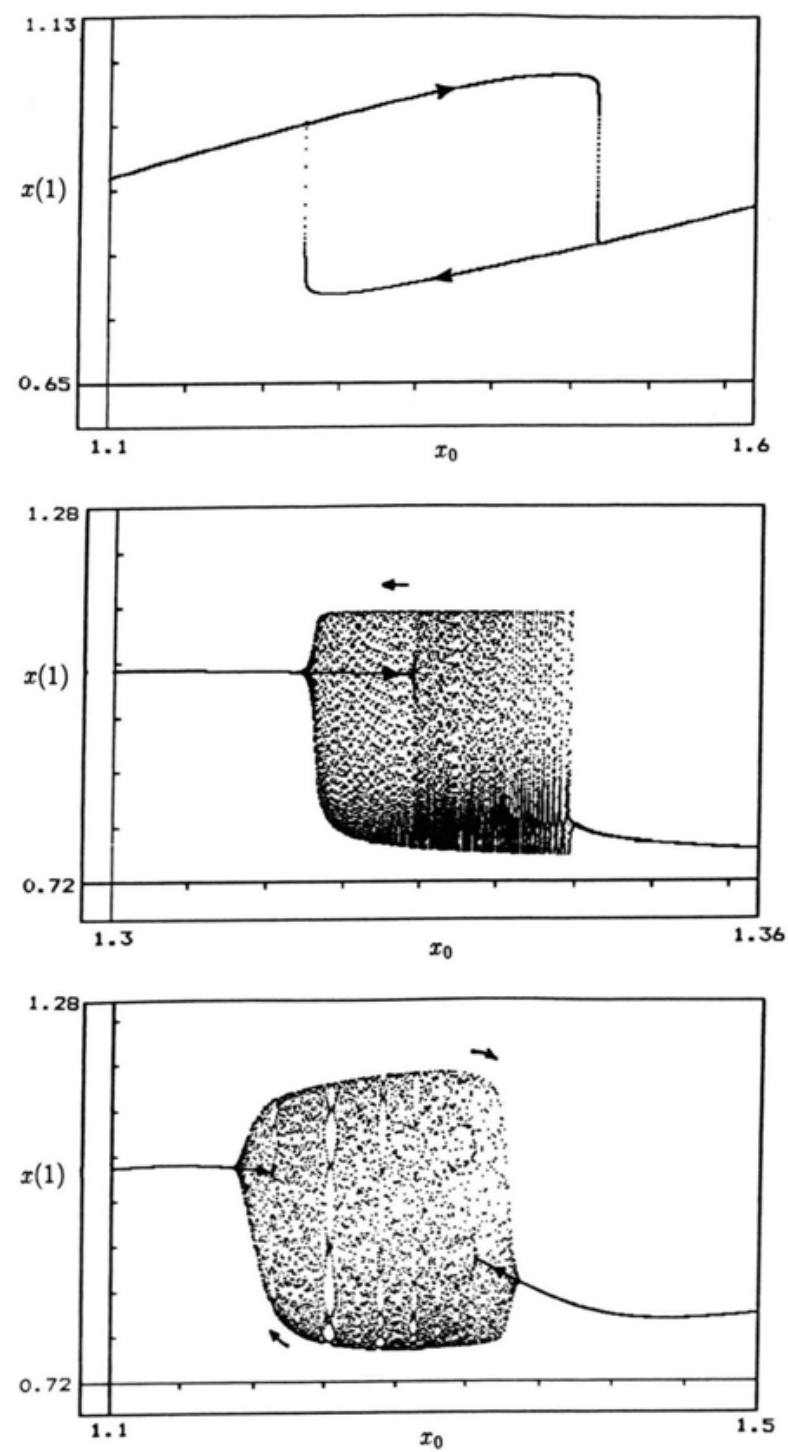

Fig. 3. Simulations of (1) $-(3)$ with constants $k_{0}=3.0, k_{1}=0.8$, $k_{2}=0.0001, k_{3}=1.5, k_{4}=2.0, k_{5}=0.15, k_{\mathrm{D}}=0.5$, and initial conditions $y(0)=0, x(1)=x_{0}$, and $y(1)=0$. Steady state value of variable $x(1)$ as a function of parameter $x_{0}$ with a) $a=1$, b) $a=2$, and c) $a=3$ (from top).

space-lattice points [5]. Here we propose a preliminary three-cell model (three space-lattice points): cell number zero describes the surface of the electrode with Nernst condition for the redox couple (benzoquinone/hydroquinone) and the production of inhibitor $y$; cell number 1 describes the diffusion layer with kinetic recovery of the benzoquinone $x$; and cell number 2 models the stock solution with constant benzoquinone concentration and zero inhibitor concentra- 
tion. This can be translated into the three-variable ODE

$$
\frac{\mathrm{d} y(0)}{\mathrm{d} t}=k_{0} x(1)-k_{4} \frac{y(0)}{k_{5}+[y(0)]^{2}}+k_{\mathrm{D}}[y(1)-y(0)],
$$

$$
\begin{aligned}
\frac{\mathrm{d} x(1)}{\mathrm{d} t}=k_{1} \frac{x_{0}-x(1)}{k_{2}+\left[x_{0}-x(1)\right]+} & k_{3}[y(1)]^{a} \\
+ & k_{\mathrm{D}}\left[x_{0}-2 x(1)\right],
\end{aligned}
$$$$
\frac{\mathrm{d} y(1)}{\mathrm{d} t}=-k_{4} \frac{y(1)}{k_{5}+[y(1)]^{2}}+k_{\mathrm{D}}[y(0)-2 \dot{y}(1)] \text {. }
$$

Here, $k_{0}$ is the rate of inhibitor production in cell zero, $k_{4}$ and $k_{5}$ describe the buffering of inhibitor, $x_{0}$ is the initial concentration of electroactive species (benzoquinone), and all diffusion coefficients are assumed to be equal.

\section{Results and Discussion}

In the following the parameters are kept constant with the exception of $x_{0}$. The dynamics of the system as a function of $x_{0}$ is studied for different exponents $a$.

For $a=1$ we find bistability and hysteresis of the variables as a function of $x_{0}$ for different initial conditions. This predicts that for one initial concentration of the electroactive species two different steady state currents can be approached, depending on the initial conditions of the variables (Figure $3 \mathrm{a}$ ).

For $a=2$ the picture is more complex. A stable steady state for low values of $x_{0}$ switches abruptly to oscillations as $x_{0}$ is increased. This oscillation then van-

[1] J. E. Frew and H. A. O. Hill, Anal. Chem. 59, 933 A (1987).

[2] G. Baier, P. Urban, and K. Wegmann, Z. Naturforsch. 44 a, 1107 (1989).

[3] G. Baier, P. Urban, and K. Wegmann, Z. Naturforsch. 43a, 987 (1988); 43a, 995 (1988). ishes into a second steady state for high $x_{0}$. Decreasing $x_{0}$, the oscillation is encountered again, this time, however, there is bistability of oscillation and the first steady state before the oscillation vanishes abruptly. This diagram indicates a subcritical Hopf bifurcation (Figure 3b).

For $a=3$ we observe oscillatory behavior with subcritical bifurcations on both sides of the window in parameter space. Note that in this case the parameter window is considerably larger than for $a=2$ (Figure $3 \mathrm{c}$ ).

The present results must be interpreted cautiously. A three-cell system describing boundary conditions and simplified kinetics of the electrocatalytic experiment with an enzyme shows bistability and simple oscillations as a function of one experimentally controllable parameter. This model is a poor approximation to Fick's second law because it neglects the continuity of the diffusion layer in the spatial dimension. It shows, however, that with fixed boundary conditions inhibition of ordinary substrate kinetics of an enzyme can lead to oscillations even in the absence of autocatalysis. Preliminary simulations show that an inhibition described by a multihumped function of inhibitor is capable of generating complex-periodic patterns of oscillations as well. This is important with respect to observations of complex and aperiodic oscillations in the experiment [2]. In addition, we would like to point out that the assumptions made are very general and should apply to a whole class of electrocatalytic experiments with minor modifications.

Paper presented at the 3rd Annual Meeting of ENGADYN, Grenoble, 1992. Thanks to all ENGADYN members for intensive discussion.

[4] R. S. Nicholson and I. Shain, Anal. Chem. 36, 706 (1964).

[5] S. W. Feldberg, in: Electroanalytical Chemistry, Vol. 3 (A. J. Bard, ed.), Marcel Dekker, New York 1969, p. 271. 